\title{
Multidimensional Hydrogen Tunneling in Supported Molecular Switches: The Role of Surface Interactions
}

\author{
Yair Litman $\odot^{*}$ \\ Fritz Haber Institute of the Max Planck Society, Faradayweg 4-6, 14195 Berlin, Germany \\ and Institute for Chemistry and Biochemistry, Freie Universität Berlin, Arnimallee 22, 14195 Berlin, Germany \\ Mariana Rossi॰ \\ Fritz Haber Institute of the Max Planck Society, Faradayweg 4-6, 14195 Berlin, Germany \\ and MPI for the Structure and Dynamics of Matter, Luruper Chaussee 149, 22761 Hamburg, Germany
}

(Received 15 May 2020; accepted 8 October 2020; published 17 November 2020)

\begin{abstract}
The nuclear tunneling crossover temperature $\left(T_{c}\right)$ of hydrogen transfer reactions in supported molecularswitch architectures can lie close to room temperature. This calls for the inclusion of nuclear quantum effects (NQEs) in the calculation of reaction rates even at high temperatures. However, computations of NQEs relying on standard parametrized dimensionality-reduced models quickly become inadequate in these environments. In this Letter, we study the paradigmatic molecular switch based on porphycene molecules adsorbed on metallic surfaces with full-dimensional calculations that combine densityfunctional theory for the electrons with the semiclassical ring-polymer instanton approximation for the nuclei. We show that the double intramolecular hydrogen transfer (DHT) rate can be enhanced by orders of magnitude due to surface fluctuations in the deep-tunneling regime. We also explain the origin of an Arrhenius temperature dependence of the rate below $T_{c}$ and why this dependence differs at different surfaces. We propose a simple model to rationalize the temperature dependence of DHT rates spanning diverse fcc [110] surfaces.
\end{abstract}

DOI: 10.1103/PhysRevLett.125.216001

Nuclear tunneling is an inherently quantum-mechanical process that can strongly impact the properties of matter in a wide variety of situations, ranging from biological enzymes to organic-based technologies [1-4]. In complex environments, it has been recognized that signatures of tunneling on rate processes are often not well captured by textbook theories [5,6] and, in particular, in hydrogen transfer reactions, the small mass of hydrogen makes tunneling pronounced [7]. Still, a theoretical description of nuclear tunneling that goes beyond a simple onedimensional approximation $[8,9]$ and considers anharmonic coupling between many degrees of freedom in larger-scale systems remains a challenge [10-12]. To build up a systematic understanding of multidimensional rate processes in the deep-tunneling regime and the impact of the environment on hydrogen dynamics, a fully $a b$ initio treatment of simple, yet nontrivial, reactions in wellcontrolled conditions is desired.

Published by the American Physical Society under the terms of the Creative Commons Attribution 4.0 International license. Further distribution of this work must maintain attribution to the author(s) and the published article's title, journal citation, and DOI. Open access publication funded by the Max Planck Society.
Tetrapyrrole macrocycles, like porphyrin, naphtalocyanine, and porphycene, show a remarkable diversity of functional properties [13-17] and were proposed, among others, as molecular switches [18-20]. The intramolecular hydrogen transfer reaction that occurs in the inner cage of these molecules, known as tautomerization, can be triggered remotely by different external stimuli [21-26]. Moreover, because the reaction takes place without a pronounced conformational change, these molecules can be incorporated in nanoscale devices. Within this area, controlling the dynamical properties of these molecules is central to advance rational design. Molecular diffusion and rotations have been more often addressed [27-31], while key aspects of the hydrogen transfer reaction mechanism and its temperature dependence remain poorly understood. This is especially due to the challenges in the description of multidimensional quantum dynamics that cannot be captured by perturbative treatments of anharmonic couplings [5,32-35].

In this Letter, we study the effect of tunneling on the double intramolecular hydrogen transfer (DHT) of two representative systems, namely, porphycene on $\mathrm{Cu}(110)$ and $\operatorname{Ag}(110)$ surfaces. In these reactions, the tunneling crossover temperature, which represents the temperature below which tunneling becomes greater than classical hopping over the barrier $\left(T_{c}=\hbar \omega_{b} / 2 \pi k_{B}\right.$, where $\omega_{b}$ is 
the imaginary frequency of the unstable mode at the transition state geometry [36]) lies close to room temperature. Specifically, we (i) identify the multidimensional DHT mechanisms at different temperatures, (ii) clarify their temperature dependence, and (iii) elucidate the role of surface fluctuations in the deep-tunneling regime. The results we obtain are able to explain puzzling experimental measurements $[4,23]$ that showed an unexpected temperature dependence of the DHT. In addition, they show that, instead of acting only as a passive observer of the reaction, in certain situations the surface takes a prominent role in the tunneling event.

We here employ a combination of density-functional theory (DFT) for the electronic degrees of freedom with the ring-polymer instanton (RPI) approximation [37,38] for the nuclear degrees of freedom. RPI can be viewed as an extension of Eyring [39] transition state theory (TST), which includes tunneling and captures anharmonic contributions along the reaction pathway. It is a semiclassical method that uses discretized closed Feynman path (CFP) integrals to evaluate tunneling rates in the deep-tunneling regime. This approximation finds the dominant stationary pathways in the CFP that connect reactants and products, the instanton pathways. Then, a series of steepest descent approximations to evaluate the flux-side correlation function leads to the instanton approximation for thermal reaction rates $k_{\text {inst }}$ [40]. The rate can be expressed as

$$
k_{\text {inst }}(\beta)=A_{\text {inst }}(\beta) e^{-S\left[\mathbf{x}_{\text {inst }}(\beta)\right] / \hbar} \text {, }
$$

where $A_{\text {inst }}$ accounts for the harmonic fluctuations around the instanton pathway $\mathbf{x}_{\text {inst }}, S$ is the Euclidean action, and $\beta=1 / k_{B} T$ with $k_{B}$ the Boltzmann constant and $T$ the temperature. In the discretized CFP space, $\mathbf{x}_{\text {inst }}$ is a firstorder saddle point. Albeit approximate, this method shows the best trade-off in situations where the quantum exponential wall would prevent a full-dimensional evaluation of the exact tunneling rate $[6,41]$.

Within the Born-Oppenheimer (BO) approximation, evaluating Eq. (1) requires the $\mathrm{BO}$ energies and forces. We here employ the most accurate level of theory affordable for the system size and number of system replicas required. We employ DFT with the Perdew-BurkeErnzerhof exchange-correlation functional [42] including the Tkatchenko-Scheffler [43] dispersion correction modified to treat physisorption on surfaces [44]. We thereby ensure a good description of the anisotropic electron density redistribution and the interfacial orbital coupling that takes place upon porphycene adsorption $[45,46]$. At this level of theory, we do not expect reaction barriers to be quantitatively accurate [47] but expect to capture qualitative trends and the correct physics. In Supplemental Material [48], we report selected geometries calculated with a rangeseparated hybrid functional for comparison and discuss the importance of van der Waals dispersion interactions in this

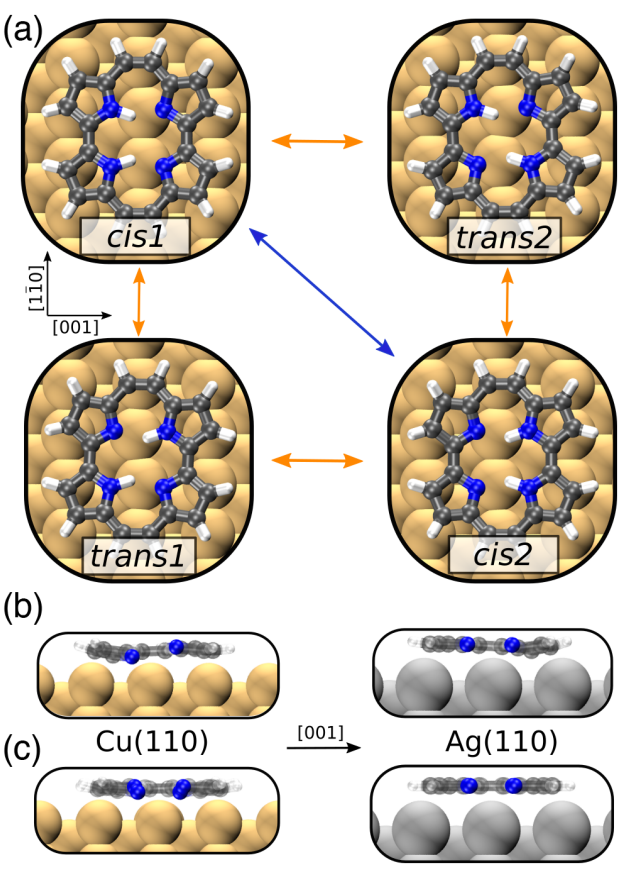

FIG. 1. (a) Top view of the local minima of porphycene on $\mathrm{Cu}(110)$. Concerted and stepwise DHT mechanisms are represented by blue and orange arrows, respectively. Lateral view of the cis (b) and trans (c) conformers, of porphycene on $\mathrm{Cu}(110)$ and $\operatorname{Ag}(110)$.

context $[58,59]$. These calculations were enabled by the combination of the FHI-aims [60] all-electron code and the i-PI $[61,62]$ universal force engine.

In porphycene, the inner-cage hydrogen atoms can adopt different configurations, giving rise to two stable tautomers, coined cis and trans. Each tautomer is twofold degenerate, and their structures on $\mathrm{Cu}(110)$ and $\mathrm{Ag}(110)$ are shown in Fig. 1. On these surfaces, the cis tautomer is more stable than the trans tautomer by 172 and $20 \mathrm{meV}$, respectively. Indeed, on the same surfaces, experiments observe almost exclusively the cis tautomer $[4,25,63]$. The DHT of the cis $\rightarrow$ cis reaction can happen through two possible mechanisms: the concerted mechanism, where the hydrogen atoms are transferred together, without the existence of a stable intermediate and passing through a second-order saddle point, and the stepwise mechanism, where the hydrogen atoms are transferred sequentially and the reaction involves a trans intermediate.

We have recently studied the DHT of porphycene in the gas phase [35] and found that there is a competition between a concerted and a stepwise DHT at different temperatures. However, new effects arise due to the interaction between the molecule and the surface. In order to rationalize them, we divide the influence of the surface on the physisorbed molecule into static and dynamical effects. The static surface effects refer to the change in the potential energy landscape upon adsorption and can affect both static and dynamical properties of the adsorbed 
(a)

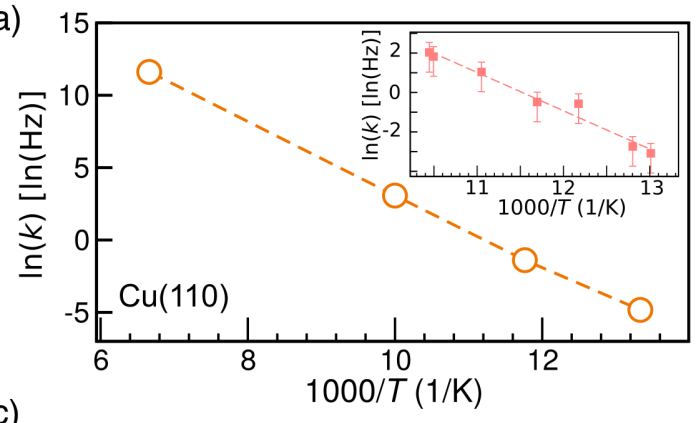

(c)

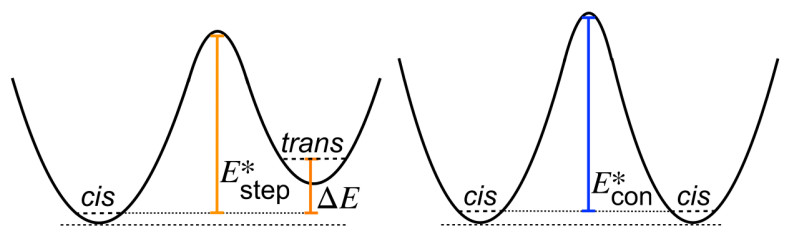

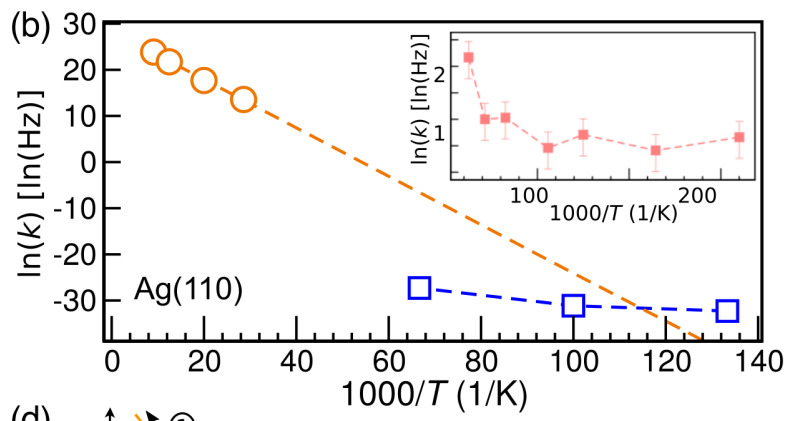

(d)

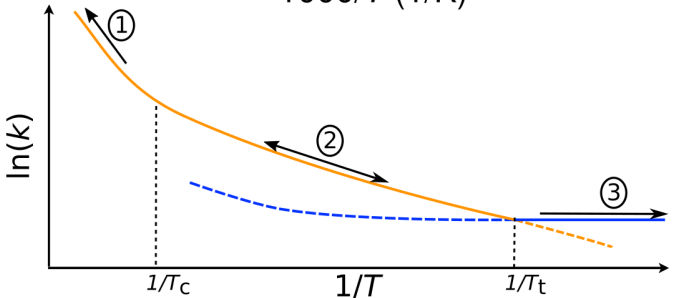

FIG. 2. (a) Calculated DHT rates $k_{\text {inst }}$ for the cis $\rightarrow$ cis reaction of porphycene on $\mathrm{Cu}(110)$ between 75 and $150 \mathrm{~K}$. At these temperatures, $k_{\text {inst }}$ includes only contributions from the stepwise mechanism. The inset shows experimental results from Ref. [23]. (b) Calculated DHT $k_{\text {inst }}$ for the cis $\rightarrow$ cis reaction of porphycene on $\operatorname{Ag}(110)$ between 7.5 and $110 \mathrm{~K}$. Orange circles represent $k_{\text {inst }}$ of the stepwise mechanism and blue squares of the concerted mechanism. The inset shows experimental results from Ref. [4]. (c) Schematic 1D potential energy surfaces of the stepwise (left) and concerted (right) reactions. The zero-point energy (ZPE) for reactant and products are shown, while the ZPE at the barrier top is not (but it was included in the multidimensional calculations). (d) Schematic representation of the different temperature dependence regimes of the stepwise (orange) and concerted (blue) DHT rate of adsorbed porphycene. Full (dashed) lines represent the dominant (minor) mechanism. The numbers 1, 2, and 3 illustrate the three different regimes; see the text.

molecule. For example, while the global minimum in the gas phase is the trans tautomer in a flat conformation, on $\mathrm{Cu}(110)$ and $\operatorname{Ag}(110)$ the relative tautomer stability is reversed and the molecule is buckled, as shown in Figs. 1(b) and 1(c). This distortion changes the DHT energy barriers and, consequently, the hydrogen dynamics. Dynamical surface effects, on the other hand, refer to the impact of the motion of the surface atoms on the molecular properties. As will be shown, they can significantly influence the dynamics of the DHT.

On $\mathrm{Cu}(110)$, we calculate $T_{c}=264 \mathrm{~K}$ for the DHT and show the instanton tunneling rates of the $c i s \rightarrow$ cis reaction in Fig. 2(a). We show rates between 75 and $150 \mathrm{~K}$, because they lie well within the deep-tunneling regime and we wish to compare with experimental results in the same temperature range [23], shown in the inset. In this case, we find a very good agreement with experiment throughout the 6orders-of-magnitude variation of the rate. The calculated effective activation energy $E_{A}$ is $190 \mathrm{meV}$. This compares well with the value of $168 \pm 12 \mathrm{meV}$ reported by experiments. Both the calculated rates and the measured ones show an Arrhenius-like temperature dependence even though tunneling is dominant at this temperature range. We proceed to explain the origin of this dependence.

The calculated DHT rates contain contributions almost exclusively from the stepwise mechanism, because at these temperatures it is several orders of magnitude faster than the concerted alternative. We propose that the trans intermediate was not observed, because its predicted residence time is $\approx 0.1 \mathrm{~ns}$, which lies beyond the time resolution of STM experiments $(\approx 100 \mu \mathrm{s})$. The instanton trajectory provides an intuitive view of the reactive process by showing the main "instantaneous" tunneling configuration that the delocalized nuclei adopt. The trajectory is visualized in Fig. S1 [48] at several temperatures. As the temperature is decreased, the reaction takes shorter pathways and crosses regions of higher PES energy. Moreover, we observe a considerable contribution from heavier atoms like $\mathrm{C}$ and $\mathrm{N}$ and, interestingly, even $\mathrm{Cu}$ atoms to the tunneling mechanism (see Table S11 [48]). These are manifestations of the multidimensional nature of the tunneling process $[10,64,65]$. They show that reducing the problem dimensionality, as previously done for porphyrin and other cyclic hydrogen-bonded solids through the BellLimbach model $[9,66]$, without at least considering a temperature dependence of the parameters, is inappropriate in this case.

After these considerations, the observed $E_{A}$ can be understood as follows. At low enough temperatures, when only the vibrational ground states (VGSs) are populated and a further decrease of the temperature does not affect the vibrational populations, the trans $\rightarrow$ cis reaction will proceed from the VGS of the reactant and will be constant with temperature. As a consequence and because of detailed balance, the inverse reaction cis $\rightarrow$ trans, which is the rate-controlling step of the stepwise mechanism, must 
TABLE I. Tunneling enhancement factor $\left(\kappa_{\text {tun }}\right)$ and kinetic isotopic effect (KIE) and surface fluctuation enhancement (SFE). See definitions and discussion in the text.

\begin{tabular}{lrrcrr}
\hline \hline Surface & $T(\mathrm{~K})$ & \multicolumn{1}{c}{$\kappa_{\text {tun }}$} & $\mathrm{KIE}_{\text {inst }}$ & $\mathrm{KIE}_{\mathrm{TST}}$ & $\mathrm{SFE}$ \\
\hline $\mathrm{Cu}(110)$ & 100 & 1.0 & 21 & 89 & 34 \\
$\mathrm{Cu}(110)$ & 85 & 1.2 & 32 & 197 & 106 \\
$\mathrm{Cu}(110)$ & 75 & 2.4 & 66 & 397 & 464 \\
$\mathrm{Ag}(110)$ & 75 & 14.9 & $\cdots$ & $\cdots$ & 4 \\
\hline \hline
\end{tabular}

show $E_{A}$ equal to the difference between the VGS energies of reactant and product, that we call $\Delta E$ [9] [see Fig. 2(c)]. The height and width of the barrier impact the absolute value of the rate, but they do not affect the Arrhenius slope in the low-temperature limit of an asymmetric reaction. Indeed, a harmonic estimation of $\Delta E$ is $172 \mathrm{meV}$, which is very close to the calculated $E_{A}=190 \mathrm{meV}$ from Fig. 2(a).

We further analyze how tunneling manifests itself in these reaction rates in Table I. A standard procedure to estimate the impact of tunneling is to compare $k_{\text {inst }}$ with the rate predicted by the Eyring TST $\left(k_{\mathrm{TST}}\right)$, since the latter neglects tunneling but includes zero-point energy (ZPE). The tunneling enhancement factor is $\kappa_{\text {tun }}=k_{\text {inst }} / k_{\text {TST }}$ [67], which is reported in Table I. Surprisingly, because these factors are close to 1 , they would seem to indicate that tunneling plays a minor role. To understand this observation, we computed the kinetic isotope effect (KIE), defined as $k^{\mathrm{H}} / k^{\mathrm{D}}$, where $k^{\mathrm{D}}$ was obtained from calculations where the inner-cage hydrogen atoms were replaced by deuterium. If tunneling were a minor effect, the only difference in these rates should be ZPE, and, since ZPE is captured by both $k_{\text {inst }}$ and $k_{\mathrm{TST}}$, the KIE of both should be similar. However, as shown in Table I, these numbers are different. TST overestimates the KIE in this particular case, for reasons outlined in Supplemental Material [48], Sec. VII. We thus conclude that $\kappa_{\text {tun }} \approx 1$ because of the following. On $\mathrm{Cu}(110)$, the $\Delta E$ (including harmonic ZPE) between reactants and products, which is a good estimate for $E_{A}$ in $k_{\text {inst }}$ as discussed above, happens to be similar to the energy difference between the ZPE-corrected reactant and transition state, which defines $E_{A}$ for $k_{\mathrm{TST}}$ (see Table II). Additionally, because close to $T_{c}$ both rates are comparable, if $E_{A}$ are similar, the prefactors also must be. This observation explains why TST fared reasonably well in these systems in the past, even without including the relevant physics of tunneling [46]. Thus, we propose that $\mathrm{KIE}_{\text {inst }} / \mathrm{KIE}_{\mathrm{TST}}$ can be an alternative measure of tunneling contributions to hydrogen transfer reactions.

The investigation of the dynamical surface effects on the DHT required RPI calculations where we fixed the surface atoms at the reactant position. The rates obtained as a result of this constrained optimization lack all contributions from fluctuations of the surface degrees of freedom. We call the ratio between the rates with and without those constraints the "surface fluctuation enhancement" (SFE). Further
TABLE II. Calculated energies ( $\Delta E$ and $\left.E_{\text {step }}^{*}\right)$, crossover temperatures $\left(T_{c}\right)$, and estimated transition temperatures $\left(T_{t}\right)$ for the DHT of porphycene at several fcc 110 surfaces. See definitions in Fig. 2 and the text. The $T_{t}$ interval is given by considering calculated or experimental references for the $\operatorname{Ag}(110)$ case (see [48]). The surface reconstruction of $\mathrm{Au}(110)$ [68] was ignored for the sake of comparison.

\begin{tabular}{lcccc}
\hline \hline Surface & $\Delta E(\mathrm{meV})$ & $T_{c}(\mathrm{~K})$ & $E_{\text {step }}^{*}(\mathrm{meV})$ & $T_{t}(\mathrm{~K})$ \\
\hline $\operatorname{Ag}(110)$ & 20 & 264 & 61 & $3-9$ \\
$\mathrm{Au}(110)$ & 59 & 264 & 85 & $8-22$ \\
$\mathrm{Cu}(110)$ & 172 & 264 & 222 & $15-41$ \\
$\mathrm{Ni}(110)$ & 199 & 297 & 347 & $13-35$ \\
$\operatorname{Pd}(110)$ & 148 & 293 & 326 & $12-34$ \\
\hline \hline
\end{tabular}

details can be found in Supplemental Material [48]. The SFEs for $k_{\text {inst }}$ on $\mathrm{Cu}(110)$ are reported in Table I and can adopt surprisingly large values. The results show that the dynamical surface effects act on the opposite direction of the static ones, increasing the tunneling rates up to 2 orders of magnitude. Interestingly, the SFEs become larger at lower temperatures, because the contribution of heavy atoms to tunneling increases with a decreasing temperature.

We then compare the $\mathrm{Cu}(110)$ with the $\operatorname{Ag}(110)$ substrate, a surface with a weaker static interaction. On $\operatorname{Ag}(110)$, porphycene is less buckled upon adsorption [see Fig. 1(b)], and the trans conformer lies only $20 \mathrm{meV}$ above $\mathrm{cis}$, but $T_{c}$ is also $264 \mathrm{~K}$. Accordingly, it was observed in experiments that the DHT rates are substantially faster on $\mathrm{Ag}(110)$ than on $\mathrm{Cu}(110)$ [4]. Unlike $\mathrm{Cu}(110)$, the measured rates show two distinct regimes [4], reproduced in the inset in Fig. 2(b). Above $\sim 10 \mathrm{~K}$ there is an Arrhenius behavior, while below $\sim 10 \mathrm{~K}$ the rate shows almost no temperature dependence. In Fig. 2(b), we show the calculated $k_{\text {inst }}$ for the stepwise and the concerted mechanisms of the cis $\rightarrow$ cis DHT of porphycene at $\operatorname{Ag}(110)$ (see calculation details in Supplemental Material [48]). We obtain an $E_{A}$ of $45 \mathrm{meV}$ for the stepwise mechanism, which compares reasonably well with the experimental $E_{A}$, which we estimate to be $12 \pm 3 \mathrm{meV}$. The calculated harmonic value of $\Delta E$ is again close to $E_{A}$ for this reaction. At $10 \mathrm{~K}$, the $\mathbf{x}_{\text {inst }}$ for the concerted mechanism starts at the reactant minimum, indicating that tunneling takes place from the reactant VGS. As such, the rates for the concerted mechanism (which is symmetric) do not change with the temperature below this point, and it becomes dominant below $8.5 \mathrm{~K}$. Hence, we can explain the two regimes observed in experiment by the change in the DHT mechanism. The lack of quantitative agreement between the calculated and measured transition temperature and rate of the concerted mechanism comes most likely from the potential energy surface used in the calculations, but some dependence of the measured rates on the STM tip cannot be fully discarded. Finally, as shown in Table I, the SFEs are smaller here than they were for $\mathrm{Cu}(110)$, accounting for a factor of 4 increase 
of the rate at $75 \mathrm{~K}$. This is consistent with the weaker adsorbate-surface interaction strength.

Building upon these considerations, the dependence of these DHT rates can be schematically understood as shown in Fig. 2(d) and predicted for other metallic fcc [110] surfaces. One needs to compute the ZPE-corrected energy difference between the cis and trans tautomers $\Delta E$, the ZPE-corrected energy barrier for the stepwise $E_{\text {step }}^{*}$, and the $T_{c}$ of the stepwise reaction. At high temperatures, the reaction behaves classically and proceeds by hopping over the lower barrier, which is normally the stepwise one, yielding a slope of $\approx E_{\text {step }}^{*}$, labeled 1 in Fig. 2(d). Considerably below $T_{c}$, the low-temperature limit of the stepwise tunneling reaction is achieved, and a slope of $\approx \Delta E$ should be observed (labeled 2). Finally, below a transition temperature $T_{t}$, the concerted mechanism becomes dominant, and the rate becomes independent of the temperature (labeled 3).

Using $\operatorname{Ag}(110)$ as a reference and building a 1D potential model for which it is necessary to calculate the barrier for the concerted mechanism $E_{\mathrm{con}}^{*}$ (see the model in Supplemental Material [48]), $T_{t}$ can be estimated for other surfaces. In Table II, the calculated values for different fcc [110] surfaces are reported, together with the corresponding estimation of $T_{t}$. All $T_{c}$ values are similar and close to $300 \mathrm{~K}$, showing the importance of tunneling at considerably high temperatures. While the estimated $T_{t}$ represent temperatures that can be achieved in different experiments especially for the stronger interacting surfaces, the resulting rates in $\mathrm{Cu}(110), \mathrm{Ni}(110)$, and $\mathrm{Pd}(110)$ would be smaller than $10^{-10} \mathrm{~Hz}$, which lies far beyond the STM detection limit.

In conclusion, we have shown how surface interactions can impact tunneling within a prototype molecular switch based on porphycene molecules adsorbed on metallic surfaces. This study was able to show that dimensionality-reduction schemes for these problems would profit from taking into account a temperature dependence in the parametrization. The counterintuitive origin of different temperature dependencies of the rates in the deep-tunneling regime and the effects of surface interaction on the dynamics of intramolecular hydrogen tunneling were also resolved. Even though full-dimensional calculations are required to get quantitative results and understand the underlying processes, we could propose a simple estimator to predict the DHT temperature dependence on different metallic surfaces.

The methodology we presented can be straightforwardly applied to other molecules on surfaces where the calculation of internal hydrogen transfer rates is sought. Limitations in the RPI approximation may arise when several local minima of the adsorbate with similar energies are present [45].

The well-defined system addressed in this work allowed us to disentangle and quantify static and dynamic effects of the environment (in this case, the metallic surface) on quantum hydrogen dynamics. We showed that dynamical effects of the environment can promote hydrogen tunneling. Such a quantification is normally not straightforward in condensed phase or biological systems. In this sense, this work shows how single-crystal substrates can be an ideal playground where cutting-edge theory and experiment can meet to provide a deeper understanding of quantum dynamics in fluctuating environments. These findings will help to address hydrogen dynamics in biology [69] and in functional materials [70], as well as guide the design and interpretation of future experiments.

The authors thank Stuart Althorpe, Aaron Kelly, and Matthias Koch for fruitful discussions and thank Takashi Kumagai and Jeremy Richardson for numerous discussions and a careful assessment of the manuscript. The authors acknowledge financing from the Max Planck Society and computer time from the Max Planck Computing and Data Facility (MPCDF) and the Swiss National Supercomputing Centre (CSCS) under Project ID No. s883.

*litman@fhi-berlin.mpg.de †mariana.rossi@mpsd.mpg.de

[1] J. P. Layfield and S. Hammes-Schiffer, Chem. Rev. 114, 3466 (2014).

[2] Y. Jiang, X. Zhong, W. Shi, Q. Peng, H. Geng, Y. Zhao, and Z. Shuai, Nanoscale Horiz. 1, 53 (2015).

[3] N. J. van der Kaap, I. Katsouras, K. Asadi, P. W. M. Blom, L. J. A. Koster, and D. M. de Leeuw, Phys. Rev. B 93, 140206(R) (2016).

[4] M. Koch, M. Pagan, M. Persson, S. Gawinkowski, J. Waluk, and T. Kumagai, J. Am. Chem. Soc. 139, 12681 (2017).

[5] J. Meisner and J. Kästner, Angew. Chem., Int. Ed. Engl. 55, 5400 (2016).

[6] M. Topaler and N. Makri, J. Chem. Phys. 101, 7500 (1994).

[7] J. T. Hynes, J. P. Klinman, H.-H. Limbach, and R. L. Schowen, Hydrogen-Transfer Reactions (Wiley-VCH Verlag, New York, 2007).

[8] D. Gerritzen and H.-H. Limbach, J. Am. Chem. Soc. 106, 869 (1984).

[9] H.-H. Limbach, J. Miguel Lopez, and A. Kohen, Phil. Trans. R. Soc. B 361, 1399 (2006).

[10] W. Fang, J. Chen, P. Pedevilla, X.-Z. Li, J. O. Richardson, and A. Michaelides, Nat. Commun. 11, 1689 (2020).

[11] J. B. Rommel, Y. Liu, H.-J. Werner, and J. Kästner, J. Phys. Chem. B 116, 13682 (2012).

[12] G. Mills, G. Schenter, D. Makarov, and H. Jónsson, Chem. Phys. Lett. 278, 91 (1997).

[13] W. Auwärter, D. Écija, F. Klappenberger, and J. V. Barth, Nat. Chem. 7, 105 (2015).

[14] A. Köbke et al., Nat. Nanotechnol. 15, 18 (2020).

[15] K. Seufert, M.-L. Bocquet, W. Auwärter, A. WeberBargioni, J. Reichert, N. Lorente, and J. V. Barth, Nat. Chem. 3, 114 (2011).

[16] T. E. Shubina, H. Marbach, K. Flechtner, A. Kretschmann, N. Jux, F. Buchner, H.-P. Steinrück, T. Clark, and J. M. Gottfried, J. Am. Chem. Soc. 129, 9476 (2007). 
[17] L. Grill, J. Phys. Condens. Matter 20, 053001 (2008).

[18] J. L. Zhang, J. Q. Zhong, J. D. Lin, W. P. Hu, K. Wu, G. Q. Xu, A. T. S. Wee, and W. Chen, Chem. Soc. Rev. 44, 2998 (2015).

[19] J. Waluk, Chem. Rev. 117, 2447 (2017).

[20] X. H. Qiu, G. V. Nazin, and W. Ho, Phys. Rev. Lett. 93, 196806 (2004).

[21] P. Liljeroth, J. Repp, and G. Meyer, Science 317, 1203 (2007).

[22] J. Kügel, M. Leisegang, M. Böhme, A. Krönlein, A. Sixta, and M. Bode, Nano Lett. 17, 5106 (2017).

[23] T. Kumagai, F. Hanke, S. Gawinkowski, J. Sharp, K. Kotsis, J. Waluk, M. Persson, and L. Grill, Phys. Rev. Lett. 111, 246101 (2013).

[24] H. Böckmann, S. Liu, J. Mielke, S. Gawinkowski, J. Waluk, L. Grill, M. Wolf, and T. Kumagai, Nano Lett. 16, 1034 (2016).

[25] J. N. Ladenthin, T. Frederiksen, M. Persson, J. C. Sharp, S. Gawinkowski, J. Waluk, and T. Kumagai, Nat. Chem. 8, 935 (2016).

[26] S. Mangel, M. Skripnik, K. Polyudov, C. Dette, T. Wollandt, P. Punke, D. Li, R. Urcuyo, F. Pauly, S. J. Jung, and K. Kern, Phys. Chem. Chem. Phys. 22, 6370 (2020).

[27] M. Eichberger, M. Marschall, J. Reichert, A. WeberBargioni, W. Auwärter, R. L. C. Wang, H. J. Kreuzer, Y. Pennec, A. Schiffrin, and J. V. Barth, Nano Lett. 8, 4608 (2008).

[28] H. Marbach and H.-P. Steinrück, Chem. Commun. 50, 9034 (2014).

[29] P. Shea and H. J. Kreuzer, J. Chem. Phys. 141, 044124 (2014).

[30] F. Buchner, J. Xiao, E. Zillner, M. Chen, M. Rckert, S. Ditze, M. Stark, H.-P. Steinrück, J. M. Gottfried, and H. Marbach, J. Phys. Chem. C 115, 24172 (2011).

[31] T. Sonnleitner, I. Swart, N. Pavliček, A. Pöllmann, and J. Repp, Phys. Rev. Lett. 107, 186103 (2011).

[32] A. Warshel, J. Phys. Chem. 86, 2218 (1982).

[33] S. Habershon, D. E. Manolopoulos, T. E. Markland, and T. F. Miller III, Annu. Rev. Phys. Chem. 64, 387 (2013).

[34] W. Fang, J. Chen, Y. Feng, X.-Z. Li, and A. Michaelides, Int. Rev. Phys. Chem. 38, 35 (2019).

[35] Y. Litman, J. O. Richardson, T. Kumagai, and M. Rossi, J. Am. Chem. Soc. 141, 2526 (2019).

[36] M. J. Gillan, J. Phys. C 20, 3621 (1987).

[37] J. O. Richardson and S. C. Althorpe, J. Chem. Phys. 131, 214106 (2009).

[38] A. Arnaldsson, Calculation of quantum mechanical rate constants directly from ab initio atomic forces, Ph. D. thesis, University of Washington, 2007.

[39] H. Eyring, Chem. Rev. 17, 65 (1935).

[40] J. O. Richardson, Int. Rev. Phys. Chem. 37, 171 (2018).

[41] J. O. Richardson, Phys. Chem. Chem. Phys. 19, 966 (2017).

[42] J. P. Perdew, K. Burke, and M. Ernzerhof, Phys. Rev. Lett. 77, 3865 (1996).

[43] A. Tkatchenko and M. Scheffler, Phys. Rev. Lett. 102, 073005 (2009).

[44] V. G. Ruiz, W. Liu, E. Zojer, M. Scheffler, and A. Tkatchenko, Phys. Rev. Lett. 108, 146103 (2012).
[45] T. Kumagai, J. N. Ladenthin, Y. Litman, M. Rossi, L. Grill, S. Gawinkowski, J. Waluk, and M. Persson, J. Chem. Phys. 148, 102330 (2018).

[46] J. Li, S. Yang, J.-C. Ren, G. Su, S. Li, C. J. Butch, Z. Ding, and W. Liu, J. Phys. Chem. Lett. 10, 6755 (2019).

[47] V. Barone, L. Orlandini, and C. Adamo, Chem. Phys. Lett. 231, 295 (1994).

[48] See Supplemental Material at http://link.aps.org/ supplemental/10.1103/PhysRevLett.125.216001 for parameters and convergence tests of electronic structure and RPI calculations, details of the model used to estimate $T_{t}$, analysis of multidimensional tunneling pathways, data related to other metallic surfaces, and extended discussion on the KIE, including Refs. [49-57].

[49] A. H. Larsen et al., J. Phys. Condens. Matter 29, 273002 (2017).

[50] J. Hermann and A. Tkatchenko, Phys. Rev. Lett. 124, 146401 (2020).

[51] Y. Litman, Tunneling and zero-point energy effects in multidimensional hydrogen transfer reactions: From gas phase to adsorption on metal surfaces, Ph.D. thesis, Freie Universität Berlin, 2020.

[52] Y. Litman, J. Behler, and M. Rossi, Faraday Discuss. 221, 526 (2020).

[53] Y. Zhang, J. B. Rommel, M. T. Cvita, and S. C. Althorpe, Phys. Chem. Chem. Phys. 16, 24292 (2014).

[54] A. Garg, Am. J. Phys. 68, 430 (2000).

[55] Y. Litman, D. Donadio, M. Ceriotti, and M. Rossi, J. Chem. Phys. 148, 102320 (2018).

[56] H. Eyring, J. Chem. Phys. 3, 107 (1935).

[57] A. V. Krukau, O. A. Vydrov, A. F. Izmaylov, and G. E. Scuseria, J. Chem. Phys. 125, 224106 (2006).

[58] M. Stöhr, T. Van Voorhis, and A. Tkatchenko, Chem. Soc. Rev. 48, 4118 (2019).

[59] G. Su, S. Yang, Y. Jiang, J. Li, S. Li, J.-C. Ren, and W. Liu, Prog. Surf. Sci. 94, 100561 (2019).

[60] V. Blum, R. Gehrke, F. Hanke, P. Havu, V. Havu, X. Ren, K. Reuter, and M. Scheffler, Comput. Phys. Commun. 180, 2175 (2009).

[61] M. Ceriotti, J. More, and D. E. Manolopoulos, Comput. Phys. Commun. 185, 1019 (2014).

[62] V. Kapil et al., Comput. Phys. Commun. 236, 214 (2019).

[63] T. Kumagai, F. Hanke, S. Gawinkowski, J. Sharp, K. Kotsis, J. Waluk, M. Persson, and L. Grill, Nat. Chem. 6, 41 (2014).

[64] M. E. Tuckerman and D. Marx, Phys. Rev. Lett. 86, 4946 (2001).

[65] C. Lin, E. Durant, M. Persson, M. Rossi, and T. Kumagai, J. Phys. Chem. Lett. 10, 645 (2019).

[66] O. Klein, F. Aguilar-Parrilla, J. M. Lopez, N. Jagerovic, J. Elguero, and H.-H. Limbach, J. Am. Chem. Soc. 126, 11718 (2004).

[67] A. N. Beyer, J. O. Richardson, P. J. Knowles, J. Rommel, and S. C. Althorpe, J. Phys. Chem. Lett. 7, 4374 (2016).

[68] W. Moritz and D. Wolf, Surf. Sci. 88, L29 (1979).

[69] J. P. Klinman and A. Kohen, Annu. Rev. Biochem. 82, 471 (2013).

[70] A. S. Tayi, A. Kaeser, M. Matsumoto, T. Aida, and S. I. Stupp, Nat. Chem. 7, 281 (2015). 